\title{
Late onset Parkinsonian syndrome in Hallervorden-Spatz disease
}

\author{
R ALBERCA, E RAFEL, I CHINCHON, J VADILLO, A NAVARRO \\ From the Departments of Neurology and Pathology, Ciudad Sanitaria Virgen del Rocĩo, Sevilla, Spain
}

SUMMARY Two siblings, from consanguineous parents, developed in their twenties a Parkinsonian syndrome. In the elder, the disease evolved for 13 years and the necropsic study was diagnostic of Hallervorden-Spatz disease. The younger sibling is severely affected after 12 years of the disorder. Several CT and one MR studies done in this patient during the last 4 years have been normal. Ultrastructural studies of the bone marrow histiocytes and blood lymphocytes disclosed peculiar inclusions. Bromocriptine in low doses proved to be a beneficial therapy for this patient.

Hallervorden-Spatz disease (HSD) is an infrequent disorder of uncertain nosology. Its morphology is characterised by axonal spheroids, deposits of ironbound pigment and neuronal loss mainly found in the globus pallidus and substantia nigra. These alterations can probably be related to a decrease in the activity of the enzyme cysteine dioxygenase in these regions. ${ }^{1}$ The disorder, usually manifested at a relatively early age, causes an extrapyramidal, mainly hyperkinetic syndrome frequently accompanied by dementia, pyramidal, and visual signs. It seldom makes its presentation after the age of 20 and in these late-onset cases a Parkinsonian syndrome may be the predominant clinical manifestation. ${ }^{2}$ It has been suggested that modern neuroimaging techniques ${ }^{34}$ or the existence of sea-blue histiocytes in the bone marrow and cytosomes in lymphocytes ${ }^{5}$ might support the clinical diagnosis of the disease. In this paper, two late-onset cases of HSD are reported and the value of these studies is discussed.

\section{Case reports}

In this family, the parents are first cousins and only two of their five children are affected. At the age of 25 the eldest sister noticed stiffness of the right foot while walking. Within 2 years the left foot was similarly affected and the following symptoms successively appeared: blank facial expression,

Address for reprint requests: Dr R Alberca. Servicio de Neurologia. CS Virgen del Rocio. Avda. Manuel Siurot s/n. 41013-Sevilla. Spain.

Received 10 October 1986 and in revised form 31 March 1987. Accepted 2 April 1987 monotonous almost inaudible voice, general stiffness and difficulty with swallowing. Examination at the age of 33 years revealed seborrhoea, amimia, absence of blinking, unintelligible speech, severe bradykinesia and generalised rigidity, dystonic posturing of the feet, exaggerated reflexes, ankle clonus and bilateral Babinski signs. She was bedridden and could not walk or eat without help. Her intelligence appeared to be unimpaired. Routine studies, including ceruloplasmin, blood and urine copper determinations and a pneumoencephalogram were normal. The patient was treated with $500 \mathrm{mg}$ of levodopa plus benserazide a day and her condition improved remarkably. However, she developed dystonic movements and a psychotic syndrome and the treatment had to be changed. She died at the age of 38. At necropsy, the globus pallidus had a slight reddish-brown discolouration and the substantia nigra had a paler appearance. Microscopy disclosed four main types of elementary lesions: axonal spheroids, iron-bound pigment, Lewy bodies and neuronal loss (fig la, b, c, d). The distribution of these changes is shown in the table. Neuronal loss and pigment were predominantly seen in the internal segment of the globus pallidus and pars reticulata of the substantia nigra. In the severely affected regions, gliosis and spongiosis accompanied the neuronal loss. Axonal spheroids were widely distributed but clearly predominated in the globus pallidus, substantia nigra and Goll and Burdach nuclei. Lewy bodies were seen in the surviving cells of the globus pallidus, substantia nigra and locus coeruleus.

At the age of 28 years, the patient's brother presented with blepharospasm that made him unable to work. The following symptoms then appeared: dystonic foot while walking, gait with short steps, slowness of movements and monotonous speech. Examination at the age of 29 showed amimia, seborrhoea, blepharospasm, severe bradykinesia, plastic rigidity in the arms, spasticity in the legs, exaggerated reflexes and bilateral Babinski sign. He walked unaided with short steps and dystonic posture of the feet. Routine studies 

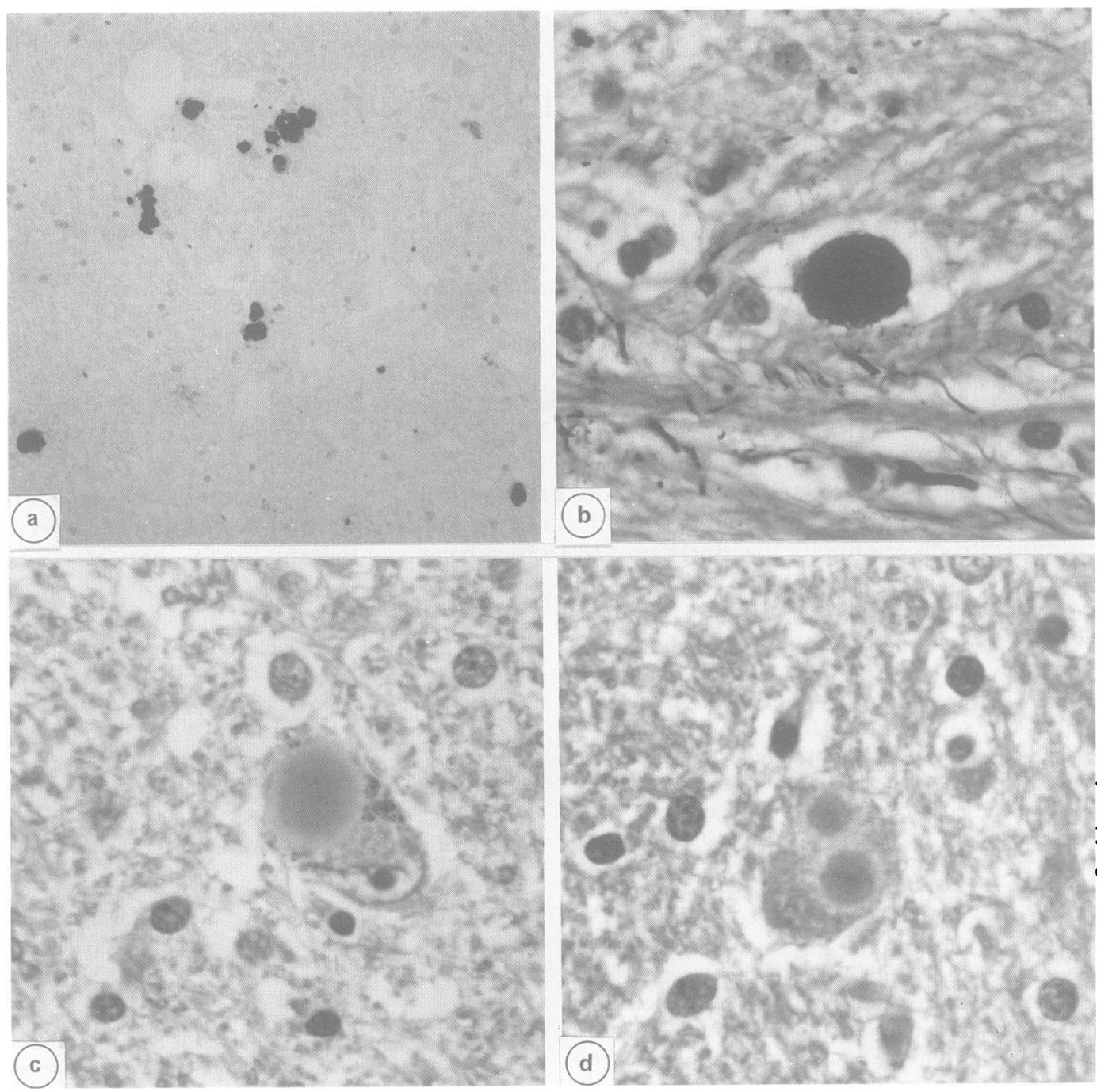

Fig.1 (a) Iron-bound pigment in the globus pallidus (Perls' prussian blue reaction $\times 65,5),($ b) Axonal swelling in the globus pallidus (Sevier-Munger silver stain $\times 250)$, (c) Lewy body in substantia nigra. (H\&E $\times 250),(d) T w o$ Lewy-like bodies in one nerve cell of the thalamus $(H \& E \times 250)$.

were normal. The patient was treated with $500 \mathrm{mg}$ of levodopa plus benserazide and $10 \mathrm{mg}$ of artane a day. His condition improved but he developed a severe psychotic syndrome with orofacial dyskinesia and the treatment was changed. At the age of 38 all medication was discontinued and the patient's condition worsened remarkably. He was bedridden, the bradykinesia was extreme, almost without spontaneous movements, and he had to be fed through a nasogastric tube. He received bromocriptine $22.5 \mathrm{mg}$ a day and since then the patient has been able to eat and walk by himself. He needs some help for daily living activities and from time to time there are psychotic episodes. The patient has been followed-up for 12 years. During the last 4 years, 0 the patient underwent four CT and one MRI studies witho normal results. The study of the bone marrow and periph eral blood lymphocytes has disclosed several abnormalities. Bone marrow stained with Giemsa-Wright showed in the 3 majority of histiocytes scanty blue cytoplasmic inclusions measuring up to $1 \mu \mathrm{m}$ in diameter. Electron microscopic study of these histiocytes showed abundant cytoplasm con $\rightarrow$ taining many inclusions of different sizes and shapes. The majority of these inclusions were evenly electron-dense whilen others were granulated and also had myelinic and vacus olated appearance. The majority of peripheral lymphocytes 0 
Table Distribution of the axonal spheroids, iron-bound pigment, Lewy bodies and neuronal loss

\begin{tabular}{lllll}
\hline & Neuronal loss & Axonal spheroids & Pigment & Lewy bodies \\
\hline Globus pallidus & +++ & +++ & +++ & + \\
Striatum & 0 & 0 & 0 & 0 \\
Thalamus & 0 & +++ & 0 & + \\
Substantia nigra & +++ & ++ & 0 & ++ \\
Amygdala & + & + & 0 & 0 \\
Red nucleus & 0 & +++ & 0 & ++ \\
Locus coeruleus & ++ & +++ & 0 & 0 \\
Cuneate nucleus & ++ & 0 & 0 & 0 \\
Gracile nucleus & 0 & 0 & 0 & 0 \\
Ambiguus nucleus & 0 & 0 & 0 & 0 \\
Cerebral cortex & 0 & 0 & 0 \\
Cerebellum & Spinal cord & & 0 & \\
\hline
\end{tabular}

The intensity of these alterations is subjectively graded from 0 (least) to +++ (most marked).

showed round inclusions of irregular electron density. Also occasionally multilaminated profiles and tubulo-reticular bodies exceeding the normal number were seen (fig 2).

\section{Discussion}

According to Dooling et $\mathrm{al}^{6} \mathrm{HSD}$ must be defined by both, morphopathological and clinical criteria. Basal ganglia are the most affected structures, specially the internal segment of the globus pallidus and the pars reticulata of the substantia nigra. Two peculiar elementary lesions, axonal spheroids and accumulation of iron-bound pigment, are found in HSD although they are not pathognomonic for this disorder. Ironbound pigment can be seen in normal aged brains and has been described in other neurological entities, including heredodegenerative and storage diseases. ${ }^{2}$ Axonal swellings are also found in many conditions and thought to be the mark of neuroaxonal dystrophies. ${ }^{2}$ What is considered to be typical of HSD is the unique combination of pallido-nigral pigmentation and widespread axonal swellings. ${ }^{2}$ The necropsy study of our patient clearly showed the typical findings described in HSD. Even the Lewy bodies found in this case have also been reported in this condition. ${ }^{6}$

HSD is clinically manifested at a relatively early age and usually presents with extrapyramidal, mainly dystonic or choreic features. In our patients, the disease appeared late, after 20 years of age, and caused a predominant Parkinsonian picture. A late-onset form of HSD has been reported in only 10 verified cases ${ }^{2}$ and at least three of them showed predominant Parkinsonian manifestations. In one of these late-onset cases an extreme decrease of dopamine in nigrostriatal areas correlated well with both, the neuronal degeneration found in the substantia nigra and the Parkinsonian syndrome of this patient. ${ }^{2}$ This dopaminergic deficiency justifies the treatment of these patients with dopaminergic medication. In our patient, bromocriptine in low doses remarkably improved his clinical condition.

Up to now, CT and MR imaging studies have failed to prove the existence of the iron accumulation typically found in HSD. ${ }^{34}$ Zones of lucency in CT studies ${ }^{5}$ and signal aberrations in $\mathrm{MRI}^{4}$ at the level of the lentiform nuclei have been described in this disorder and these findings could help in the diagnosis of the disease. In our case, CT and MR studies were normal and in another referred late-onset case, ${ }^{2}$ the CT only showed enlargement of the ventricles and cortical atrophy. It could be that in these late-onset cases the pallidonigral lesions are not severe enough to be detected in these studies.

Extracerebral morphopathological manifestations of HSD have been rarely reported. Ultrastructural studies of the skin have disclosed peculiar electrondense, membrane bound intracellular material of unknown origin. ${ }^{7}$ Sea-blue histiocytes in the bone marrow and several kinds of cytosomes in peripheral lymphocytes were found in an assumed case of HSD. ${ }^{5}$ In our patient, Giemsa-Wright staining of the bone marrow showed blue inclusions in almost every histiocyte but in number significantly lower than what is expected in typical sea-blue histiocytes. However, in the ultrastructural study both types of inclusions had a similar appearance. In addition, numerous cytosomes were seen in peripheral lymphocytes. These findings are unspecific and may be seen in many conditions, ${ }^{25}$ among them ceroid lipofuscinosis and dystonic lipidosis. In our patient, all these disorders were confidently ruled out. These changes are possibly related to the main pathological mechanism acting in HSD and its demonstration may help in the diagnosis of the condition.

According to the review of Dooling et $a l^{6}$ most cases of HSD start before the age of 20 years and dystonic or choreic features clearly predominate in 

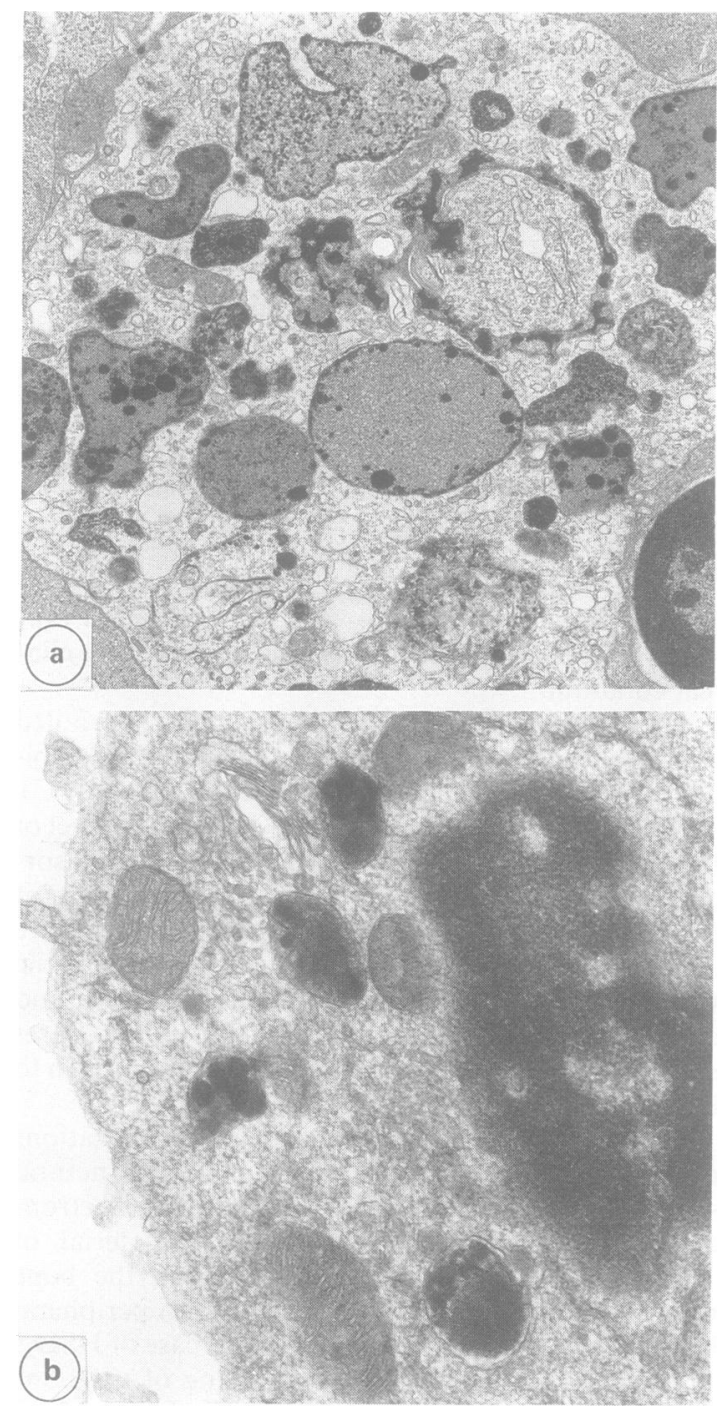

Fig. 2 Top: Detail of histiocytes showing variegated inclusion bodies of different density and shapes: Bottom: Detail of lymphocytes with heterogeneous electron dense inclusions. $(\times 20,000)$. these early-onset observations. In the unusual late $\underset{\infty}{Z}$ form, the disease appears after 20 years of age and $\mathrm{a} \cong$ relatively high proportion of these cases start with क predominant Parkinsonian manifestations. ${ }^{2}$ It is difficult to explain this variability in the clinical 0 presentation and also in the pathological findings of HSD. It probably depends partly on the structure $\stackrel{5}{=}$. firstly involved and the stage of evolution at which the necropsy is done. However, this variability could also. be explained by the heterogeneous nature of the cases $\overrightarrow{\vec{F}}$ today included in the diagnosis of HSD. The disorder is genetically determined ${ }^{1}$ and our family clearly shows the autosomal recessive transmission rarely $\frac{\bar{D}}{\bar{D}}$ demonstrated in the late-onset form. Different genetic $\overrightarrow{\widetilde{\phi}}$ and enzymatic errors could also explain the differences among patients with HSD. ${ }^{1}$

\section{References}

1 Perry TL, Norman MG, Tong VW, et al. Hallervorden- $\vec{N}$ Spatz disease: cysteine accumulation and cystein $\vec{\sigma}$ dyoxygenase deficiency in the globus pallidus. $A m$ of Neurol 1985;18:482-9.

2 Jankovic J, Jirpatrick JB, Blomquist KA, Langlais $\mathrm{P} \frac{\text { }}{\text {, }}$ Bird ED. Late-onset Hallervorden-Spatz disease presenting as familial Parkinsonism. Neurology 1985; 35:227-34.

3 Dooling EC, Richardson EP, Davis KR. Computee tomography in Hallervorden-Spatz disease. Neurolog 1980;30:1128-30.

4 Littup PJ, Gebarski SS. MR imaging of Hallervorden- $\vec{\varphi}$ Spatz disease. J Comp Assist Tomogr 1985;9:491-3.

5 Swaiman KF, Smith SA, Trock GL, Siddiqui AR. Seablue histiocytes, lymphocytic cytosomes, movement disorder and $59 \mathrm{Fe}$ uptake in basal ganglia: Hallervorden-Spatz disease or ceroid disease with abnormal isotope scan? Neurology 1983;33:301-5.

6 Dooling EC, Schoene WC, Richardson EP. \& Hallervorden-Spatz syndrome. Arch Neurol $\overrightarrow{\vec{O}}$ 1974;30:70-83.

7 Stover ML, Zimmerman AW, Donaldson JO. Skin ultrastructural changes in Hallervorden-Spatz syndrome. Neurology 1981;31:93. 